\title{
¿ES LA CONCIENCIA UN ESTADO DE LA MATERIA?
}

\author{
Francisco José Soler Gil \\ Universidad de Sevilla
}

\begin{abstract}
Resumen: En este artículo se resumen las líneas generales del programa de investigación que propone considerar la conciencia como un estado particular de la materia, se identifica su supuesto inicial, y se discuten dos posibles objeciones a este programa. Se concluye que la concepción de la conciencia como un estado de la materia no es capaz de explicar adecuadamente el papel de la conciencia en los procesos de toma de decisiones.
\end{abstract}

Palabras clave: conciencia; libertad; dualismo; materia; indeterminismo; perspectiva objetiva; perspectiva subjetiva.

\section{Is consciousness a state of the matter?}

Abstract: This article summarizes the general lines of the research program that proposes to consider consciousness as a particular state of matter, identifies its initial assumption, and discusses two possible objections to this program. We conclude that the conception of consciousness as a state of matter is not capable of adequately explaining the role of consciousness in decision-making processes. Keywords: consciousness; freedom; dualism; matter; indeterminism; objective perspective; subjective perspective.

Recibido: 05/10/2017 Aprobado: 5/11/2017 


\section{Introducción}

El título de esta breve reflexión alude, entre signos interrogativos, al título de un artículo relativamente reciente del físico Max Tegmark: «La conciencia considerada como un estado de la materia» (Tegmark, 2015). No porque dicho artículo sea especialmente relevante, sino más bien debido a que es un título claro, y sobre todo debido a que se trata de un ejemplar típico de las publicaciones que están surgiendo en el entorno de una de las líneas por las que se intenta abordar actualmente el proyecto de la explicación científica de la conciencia.

El artículo técnico principal de esta línea de investigación se debe al neurocientífico Giulio Tononi: «La conciencia como información integrada: Un manifiesto provisional»(Tononi, 2008). Deuda que el propio Tegmark no tiene ningún inconveniente en reconocer ya en la segunda página de su texto.

Pero si queremos encontrar las reflexiones de fondo requeridas para hacer frente a las objeciones filosóficas más inmediatas al proyecto, tendremos que retrotraernos aún más, al libro de Gerald Edelman y Giulio Tononi «El universo de la conciencia. Cómo la materia se convierte en imaginación» (Edelman, Tononi, 2002). 
En el mínimo espacio de la presente reflexión no se puede seguir este planteamiento en detalle ${ }^{1}$, pero trataré de apuntar al menos algunas ideas clave para entender el significado, el alcance y los posibles problemas del mismo. En concreto, y por este orden, me voy a referir a: (a) El supuesto inicial decisivo; (b) qué estados de la materia serían conscientes; (c) cómo se afronta desde esta perspectiva la primera objeción que surge, la objeción del «error categorial»; y finalmente (d) el problema que tal vez podría resultar más serio de este planteamiento: el problema de la irrelevancia de la perspectiva subjetiva.

\section{E1 supuesto inicial}

Lo que se pretende, en un programa de investigación dedicado a la conciencia como un estado de la materia es, obviamente, explicar en qué consiste la conciencia, en qué condiciones se presenta, y por qué posee determinadas características. Pero no tendría sentido adoptar este enfoque, y buscar los rasgos de los estados particulares conscientes de la materia, si se pensara que hay aspectos esenciales (o en algún sentido relevantes) de la conciencia que son inmateriales. Por eso, Tegmark resume el punto de partida del programa así:

1 Los planteamientos de base del programa de investigación de la conciencia que vamos a considerar aquí, además de los de otros programas, han sido expuestos y discutidos con más detalle en (Arana, 2015). 
$\mathrm{El}$ supuesto clave de este artículo es que la conciencia es una propiedad de ciertos sistemas físicos, sin ninguna "salsa secreta", o elementos no físicos. [...] El lugar de partir del problema duro de por qué una disposición de partículas puede sentirse consciente, nosotros partimos del hecho duro de que ciertas disposiciones de partículas (tales como su cerebro) se sienten conscientes, mientras que otras (como por ejemplo su almohada) no lo hacen, y preguntamos qué propiedades de la disposición de las partículas generan la diferencia (Tegmark, 2015: 2).

Formulado con otras palabras, el mismo supuesto lo encontramos casi al principio de la obra básica de Edelman y Tononi:

La suposición física asevera que bastan los procesos físicos convencionales para construir una explicación científica adecuada de la conciencia: o sea, que no se permite el dualismo. En particular, hemos de suponer que la conciencia es una forma especial de proceso físico que surge de la estructura y dinámica de ciertos cerebros (Edelman, Tononi, 2002: 26).

Por tanto, la apuesta consiste en que la física, con sus partículas y fuerzas descritas por las teorías usuales, nos proporciona todas las herramientas conceptuales necesarias para construir una explicación completa y adecuada sin que falte ninguna "salsa secreta" - de la conciencia.

Y el programa se desarrolla, por tanto, tratando de identificar qué rasgos tienen que poseer los procesos físicos para que se trate de procesos físicos conscientes. Y cómo esos rasgos físicos explican los distintos aspectos de nuestra experiencia consciente. 


\section{Características de los estados de materia conscientes}

En una primera variante, centrada exclusivamente en el cerebro humano, Edelman y Tononi propusieron la llamada «hipótesis del núcleo dinámico», según la cual la conciencia es un proceso físico que se da en el cerebro, pero no en una parte determinada del mismo, sino en estados determinados por agrupaciones cambiantes de un número grande de neuronas (pero siempre muchas menos que el conjunto total de neuronas del cerebro). Más concretamente:

1. Un grupo de neuronas puede contribuir directamente a la experiencia consciente sólo si forma parte de una agrupación funcional distribuida que, a través de interacciones de reentrada en el sistema talamocortical, alcanza un alto grado de integración en unos centenares de milisegundos.

2. Para sustentar la experiencia consciente, es esencial que esta agrupación funcional esté altamente diferenciada, es decir, que presente valores altos de complejidad. (Edelman, Tononi, 2002: 177).

Más adelante, en su artículo de 2008, Tononi reelabora la segunda condición, para referirse a la alta diferenciación de los estados conscientes en términos de información. Haciendo esto, puede generalizar la posibilidad de conciencia a todos los sistemas de procesamiento de información que cumplan con dos condiciones que Tegmark (comentando el mencionado artículo de Tononi) resume así: 
1. Información: Tiene que darse un gran repertorio de estados accesibles, es decir, habilidad para almacenar una gran cantidad de información.

2. Integración: Esta información debe estar integrada en un todo unificado, es decir, debe ser imposible descomponer el sistema en partes casi independientes, porque, de otro modo, cada una de esas partes se sentiría subjetivamente como conciencias separadas (Tegmark, 2015:2).

\section{4. ¿Estamos ante un error categorial?}

Leyendo textos como los que acabo de citar, uno puede fijarse en aspectos diversos. Uno de ellos es que, al tratar de la conciencia, se la está describiendo en términos de procesos físicos en los que tales o cuales componentes materiales (por lo común neuronas) son durante un tiempo integrados en una unidad dinámica caracterizada por interacciones mutuas de naturaleza eléctrica.

Lo cual puede conducir a una primera objeción al programa: ¿cómo vamos a explicar algo como la experiencia subjetiva de ser consciente por medio de piezas conceptuales que no tienen nada que ver con lo subjetivo? ¿ $\mathrm{No}_{0}$ estamos incurriendo aquí en un error categorial?

Leibniz expuso el problema de un modo clásico por medio de su famoso «argumento del molino»:

[...] imaginémonos que haya una máquina cuya estructura la haga pensar, sentir y tener percepción; podremos concebirla agrandada, conservando las mismas proporciones, de tal manera que podamos entrar en ella como en un molino. Esto 
supuesto, si la inspeccionamos por dentro, no hallaremos más que piezas que se impelen unas a otras, pero nunca nada con que explicar una percepción (Leibniz, 2001: 109).

Y también John Locke se refirió al asunto, desde otro ángulo:

Un hombre ciego y estudioso que se había exprimido los sesos para comprender los objetos visibles, y que había utilizado todas las explicaciones de sus libros y de sus amigos para comprender el significado de la luz y de los nombres de los colores que tan a menudo encontraba, se jactaba un día de que por fin había logrado comprender el significado del color escarlata. A lo que un amigo le preguntaba qué es el color escarlata, y él le responde: "es como el sonido de una trompeta" (Locke, 1975: 389).

¿No estaríamos, pues, incurriendo aquí en el mismo error de intentar explicar algo por medio de conceptos que no se aplican en el ámbito de la realidad donde se encuentra el objeto que queremos explicar?

La respuesta que autores como Edelman y Tononi han ensayado a esta objeción se basa en indicar que una cosa es explicar qué sea la conciencia, y otra cosa es cómo sentimos cada uno de nosotros subjetivamente nuestra conciencia:

Podemos describir el agua con el lenguaje ordinario, pero podemos igualmente describir el agua, al menos en principio, en términos de átomos y leyes de la mecánica cuántica. Lo que hacemos es conectar dos niveles de descripción de la misma entidad externa —uno común y otro científico de extraordinario poder 
explicativo y predictivo. [...] [Pero] en el caso de la conciencia somos lo que describimos científicamente. Esta aseveración es un reconocimiento de la especial condición epistémica de la conciencia. Si la aceptamos [...] todavía podremos intentar proporcionar una explicación científica satisfactoria de la conciencia del mismo modo que lo hacemos respecto de cualquier otro sujeto científico: qué tipo de proceso físico es, por qué tiene las propiedades que tiene y en qué condicondiciones se produce. [...] [En cambio] los aspectos subjetivos o cualitativos de la conciencia, al ser privados, no pueden ser comunicados directamente por medio de una teoría científica que, por su propia naturaleza, es pública e intersubjetiva (Edelman, Tononi, 2002: 24-27).

Por tanto, lo que buscaría el programa de estudio de la conciencia como un estado de la materia es explicar en qué consisten objetivamente los estados conscientes, dejando de lado las vivencias subjetivas de los mismos, que resultan invisibles, por privadas, para la ciencia.

Ciertamente, este planteamiento desactiva, hasta cierto punto, la objeción leibniziana, y legitima el proyecto de ofrecer una descripción de los estados conscientes, tal y como estos se contemplan desde el plano físico. Sin embargo, ¿podemos decir realmente que una descripción así es una explicación de la conciencia? ¿En qué sentido y hasta qué punto?

\section{El problema de la irrelevancia de la perspectiva subjetiva}

Desde luego, hay aspectos de la experiencia consciente que parece que sí pueden (o podrían en principio) ser explicados por medio del programa que 
estamos considerando. Por ejemplo, el hecho de sólo experimentemos un estado consciente cada vez, su duración, y que este estado no pueda ser dividido en partes, etc.

Sin embargo, los promotores del programa de la conciencia como un estado de la materia van más allá de eso, y lo que sugieren es que sus planteamientos:

...explican realmente las propiedades fundamentales de la conciencia, en lugar de buscar simples correlatos de ellas (Edelman, Tononi, 2002: 31).

O, como mencionaba al principio del artículo: que no hay «ninguna "salsa secreta", o elementos no físicos», «que bastan los procesos físicos convencionales para construir una explicación científica adecuada de la conciencia». O, dicho de otro modo: que no queda nada relevante fuera de su explicación.

Quedaría fuera, sí, la percepción subjetiva de los estados de conciencia. Pero algo así no parece, en estos autores, tener mayor importancia.

Ahora bien, ¿ ¿puede resultar verosímil un enfoque así, en el que una comprensión completa de la conciencia puede lograrse al margen de la vivencia subjetiva de los estados de conciencia?

A mi modo de ver, no. Y un ejemplo concreto tal vez baste para mostrar por qué no:

Supongamos que una persona se ve en la circunstancia de tener que tomar una decisión complicada. En los tiempos que corren, se entenderá bien esta circunstancia si la concretamos en un funcionario que, habiendo descubierto en su departamento un caso grave de corrupción, en el que se encuentran 
implicados algunos de sus superiores, se plantea si debe denunciar publicamente los hechos o no. En su conciencia tendrá lugar una deliberación, en la que serán sopesados todo tipo de argumentos a favor y en contra de la denuncia: El deber moral de enfrentarse a la corrupción frente a las desventajas que ese enfrentamiento puede conllevar para la propia carrera, incluyendo una eventual pérdida del empleo. La complicidad que supondría no actuar frente al temor a ser considerado entre sus compañeros como un delator. Los efectos beneficiosos de todo tipo que se derivan de la persecución de ciertos delitos frente a las quizás remotas posibilidades de que la justicia intervenga de hecho para perseguirlos, etc. etc.

Finalmente, tras un periodo de reflexión más o menos largo, concluirá que los argumentos en favor de una de las partes de este juicio son más fuertes, y tomará una decisión. En un sentido o en otro. Y ni él ni nosotros dudaríamos probablemente en decir que esa decisión no es aleatoria, sino que está determinada por el proceso reflexivo que la precede. Es decir, la decisión queda determinada por la deliberación previa, y se explica apelando a dicha deliberación.

Ahora bien, al describir este proceso, y su desenlace final, he estado empleando hasta ahora la perspectiva subjetiva: la forma en que la conciencia percibe «desde dentro» lo que ocurre. Sin embargo, si consideramos el mismo desarrollo desde la perspectiva de la descripción objetiva física, que, según los autores del programa explicativo que estamos considerando, es una descripción que no deja nada relevante fuera, nos veremos obligados a concluir que el proceso y la decisión resultante han sido en realidad aleatorios. 
Esto es así porque, como han puesto de manifiesto diversos autores pero encontramos ante todo analizado con gran detalle en (Falkenburg, 2012) - , los procesos físicos que tienen lugar en el cerebro son esencialmente indeterministas. Al menos si aceptamos el marco que nos proporciona la física actual -que es lo único que cabe hacer, so pena de abandonarnos a especulaciones arbitrarias-.

Con lo que nos encontramos ante la situación de que reducir la conciencia a un estado de la materia — que correspondería a su «descripción objetiva»—, nos conduce a considerar como aleatorio un resultado que sabemos - si tomamos en cuenta la «descripción subjetiva»— que en modo alguno es tal.

A mi modo de ver, la conclusión que se sigue de lo expuesto es que tomar la conciencia como un estado de la materia es un punto de partida que conduce a un modelo de la misma que, si bien puede llegar a explicar algunos de sus rasgos, resulta incapaz de dar cuenta lo que posiblemente sea su actividad más importante: su papel en la toma de decisiones. En definitiva, su papel como fundamento de la libertad humana.

\section{Referencias}

J. Arana, La conciencia inexplicada, Madrid, Biblioteca Nueva, 2015.

G. Edelmann, G. Tononi, El universo de la conciencia, Barcelona, Crítica, 2002.

B. Falkenburg, Mythos Determinismus, Berlin, Springer, 2012.

G. W. Leibniz, Monadología, Madrid, Biblioteca Nueva 2001.

J. Locke, An Essay Concerning Human Understanding, Oxford, Clarendon Press, 1975. 


\section{Francisco José Soler}

M. Tegmark, "Consciousness as a State of Matter" en: Chaos, Solitons E Fractals, 2015 (76), pp. 238-270

G. Tononi, "Consciousness as integrated information: a provisional manifesto" en: Biol. Bull., 2008 (215)3 pp. 216-42.

Francisco Soler Gil

soler@unibremen.de 